\title{
La espiral de precariedad: desempleo, temporalidad y parcialidad involuntaria en la población joven, 2005-2021
}

\author{
The spiral of precariousness: unemployment, temporary and \\ involuntary part-time in the young population, 2005-2021
}

\author{
Margarita Torre* \\ Universidad Carlos III de Madrid \\ ORCID ID: 0000-0002-1051-9717
}

Recibido: $25 / 6 / 2021$

Aceptado: 28/6/2021

doi: https://doi.org/10.20318/labos.2021.6223

La inserción en el mercado de trabajo es una etapa clave en el ciclo vital de las personas. Estar desempleado o subempleado a una edad temprana puede tener un "efecto cicatriz" en las trayectorias profesionales de los jóvenes, que acabarán enfrentándose a niveles salariales más bajos, menos oportunidades de desarrollo profesional y, en última instancia, pensiones más bajas (OCDE 2016). Los primeros años en el mercado de trabajo de trabajo son, además, cruciales cuando se trata de tomar decisiones trascendentes, como el abandono del hogar familiar y la formación de familia (Chinchilla et al. 2017). Con la crisis global del 2008 se produjo un fuerte incremento del desempleo, un estancamiento en el crecimiento de los salarios y un aumento de la brecha en las habilidades adquiridas y las empleadas en el trabajo, particularmente entre la población más joven (Berry y McDaniel 2020). La reciente emergencia sanitaria por COVID-19 supone un nuevo revés para la sociedad, si bien las repercusiones económicas y sanitarias de la pandemia han sido asimétricas entre los distintos grupos de edad. La población joven corre menos riesgo de desarrollar síntomas graves de salud física relacionados con la COVID-19 que las cohortes de mayor edad. Sin embargo, la recesión económica provocada por los confinamientos les sitúa en una trayectoria incierta a la hora de encontrar y mantener empleos e ingresos de calidad (OECD 2020).

Ante esta realidad, las organizaciones internacionales alertan del incremento de una brecha intergeneracional (OCDE 2020). Los nacidos entre 1985 y 1995 se posicionan en una posición particularmente vulnerable, sufriendo las consecuencias de dos recesiones económicas en un momento clave de su formación y acceso al empleo hasta el punto de que hay quien habla de la generación perdida entre dos crisis. A esto contribuye que los regímenes de bienestar europeos -los cuales incluyen el mercado laboral, sistemas de protección social y redes de apoyo familiares- no están cumpliendo con el principio básico de justicia intergeneracional, según el cual todos los grupos de edad deben disfrutar de niveles equivalentes de bienestar económico (Fernández 2017). En consecuencia, el bienestar de los jóvenes y las generaciones futuras suponen un reto de carácter urgente.

En las páginas que siguen examinaremos, de manera concisa, la evolución del empleo juvenil entre 2005 y 2021. Concretamente, nos centraremos la evolución del desempleo, el trabajo tempo-

*mtfernan@clio.uc3m.es 
ral y la falta de horas de trabajo. A continuación, examinaremos el impacto del confinamiento en la población joven. El artículo concluye con una breve reflexión sobre la situación de la precariedad juvenil y la necesidad apremiante de reformas estructurales.

\section{Los últimos en llegar, los primeros en irse: evolución del desempleo 2005-2015}

La Figura 1 muestra la evolución del desempleo entre 2005 y 2021 para diferentes grupos de edad. En primer lugar, observamos que el desempleo es significativamente mayor en la población joven, particularmente entre los menores de 25. Esto confirma que las dificultades de encontrar un trabajo son mayores para quienes abandonan el sistema educativo a edades muy tempranas (Requena 2016, OECD 2015). En segundo lugar, el porcentaje de desempleados aumentó drásticamente en todos los grupos de edad con el estallido de la crisis global de 2008. Las cifras de desempleo alcanzan valores máximos en 2013, llegando al 56\% en la población de menos 25 años, 34,4\% para los que tenían edades comprendidas entre 25 y 29 y $22,7 \%$ para el resto de los trabajadores. A partir de ese momento, coincidiendo con la etapa de recuperación económica, el paro desciende de manera sostenida hasta el ańo 2019. Finalmente, en 2020, se produce un cambio de tendencia como consecuencia de los confinamientos derivados de la emergencia sanitaria. Entre enero y marzo de 2021, aproximadamente el $40 \%$ de los jóvenes menores de 25 que buscaban empleo no lo encontraba (frente al 33\% de 2019). Para quienes tienes tenían entre 25 y 29 años, la cifra pasó del 18,6\% al $24 \%$. Entre los mayores de 30 años el incremento fue algo menor, de 1,4 puntos porcentuales. En general, los números constatan que los jóvenes son los últimos en entrar al mercado de trabajo, pero son los primeros en salir cuando hay crisis.

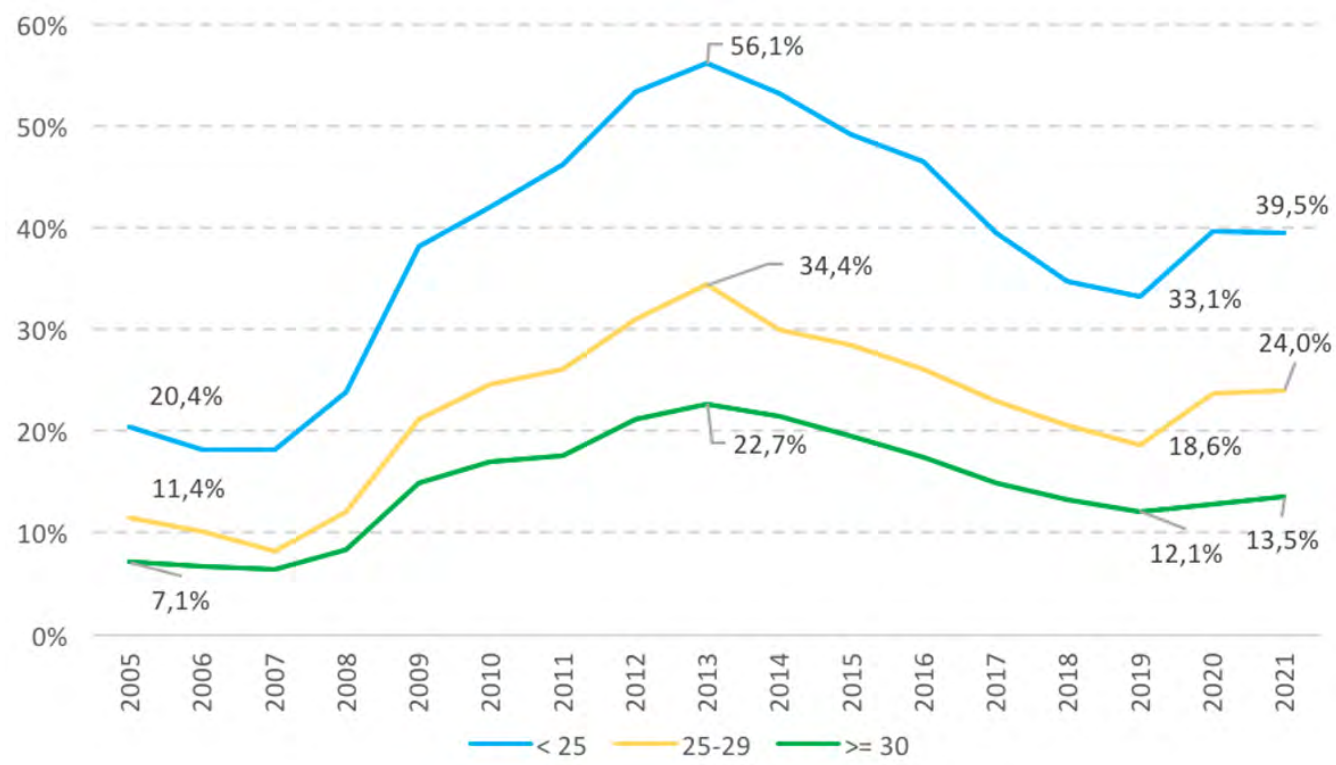

Figura 1. Porcentaje de población desempleada por tramos de edad, 2005-2021

Fuente: Elaboración propia a partir de datos de EPA 2005-2020 (T2) y 2021 (T1)

Con estas cifras, España encabeza la lista de paro juvenil en Europa, lejos del 17,2\% registrado en la eurozona el pasado marzo (Eurostat 2021). Además, esta es sólo una cara de moneda, ya que gran parte del empleo juvenil es un empleo precario tanto en términos de estabilidad como de cantidad de horas trabajadas. Las secciones a continuación examinan estas dos cuestiones. 


\section{La doble precariedad: empleo temporal y falta de horas de trabajo}

En un número anterior de esta misma revista, Daniel Prados (2021) discutía los efectos de las tasas de temporalidad en España. De acuerdo con el autor, las altas y persistentes tasas de temporalidad en España tienen consecuencias negativas tanto en términos de equidad como de eficiencia económica. Según evidencia Prados, aunque la contratación temporal ha venido siendo utilizada desde los 80 como una forma de política de empleo, su uso no ha contribuido a generar más puestos de trabajo. Además, la contratación temporal lleva aparejada una remuneración más baja y los trabajadores temporales reciben menos formación en el trabajo (Cabrales, Dolado y Mora 2017), lo cual puede tener un impacto negativo en la productividad presente y futura de los trabajadores. En lo que respecta al plano individual, el aumento de la temporalidad contractual de los empleos juveniles ha hecho más difícil emanciparse y establecer nuevas unidades familiares (Requena 2006).

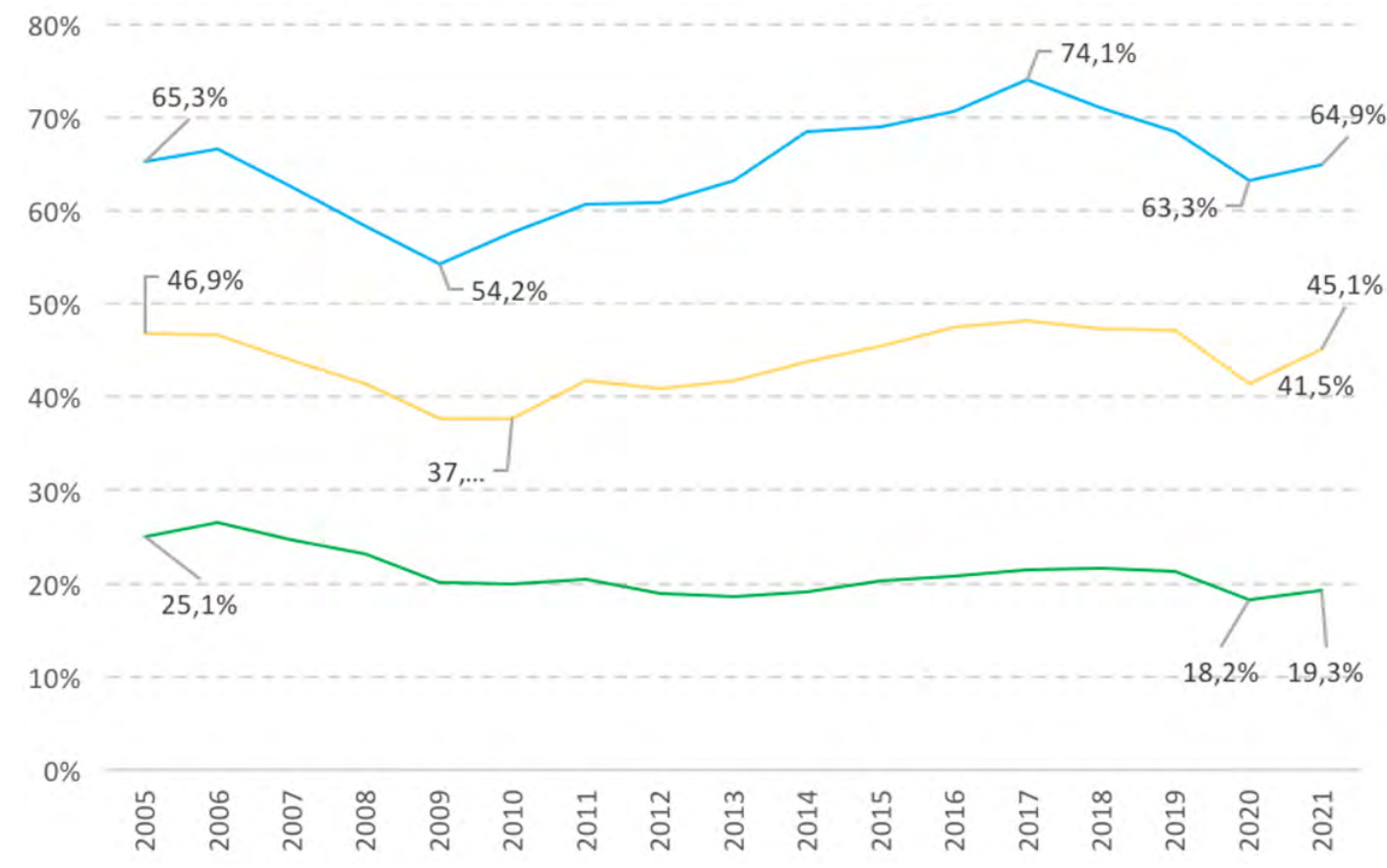

Figura 2. Evolución de la temporalidad por grupos de edad, 2005-2021.

Fuente: Elaboración propia a partir de datos de EPA 2005-2020 (T2) y 2021 (T1)

La Figura 2 muestra la evolución del empleo temporal entre 2005 y 2021 para tres grupos de edad. Al igual que sucedía con el desempleo, son los más jóvenes quienes sufren unas tasas de temporalidad más elevadas, llegando al 74\% (grupo 16-24) y 48\% (grupo 26-29) en el año 2017. Entre la población adulta el empleo temporal tiene su máximo en el año 2006 (26,6\%), decrece hasta 2009 y, a partir de ahí, se mantiene relativamente constante durante el resto del período. Son los mayores, además, quienes menos han acusado el confinamiento.

$\mathrm{Al}$ riesgo permanente que supone encadenar empleos temporales hay que sumar otro gran problema para el desarrollo profesional: la falta de horas de trabajo. En la Figura 3 aparece representado el porcentaje de población empleada a tiempo parcial según género y grupo de edad. La figura arroja dos resultados particularmente interesantes. Por un lado, la contratación a tiempo pacial es mayor entre las mujeres que entre los hombres, independientemente de su edad. Las mujeres de 30 o más años tienen cifras de parcialidad superiores a las de los hombres jóvenes, si bien esa diferencia 
se ha ido estrechando en el tiempo. Por otro lado, dentro de cada género, el empleo a tiempo parcial es más común entre los jóvenes que entre los adultos. La población joven, además, han sufrido un incremento más evidente durante el confinamiento, pasando de $29 \%$ a de $34 \%$ las mujeres y de $17,6, \%$ a $19,5 \%$ los hombres entre el segundo trimestre de 2020 y el primero de 2021 . El impacto del confinamiento ha sido menor para los mayores de 30 años.

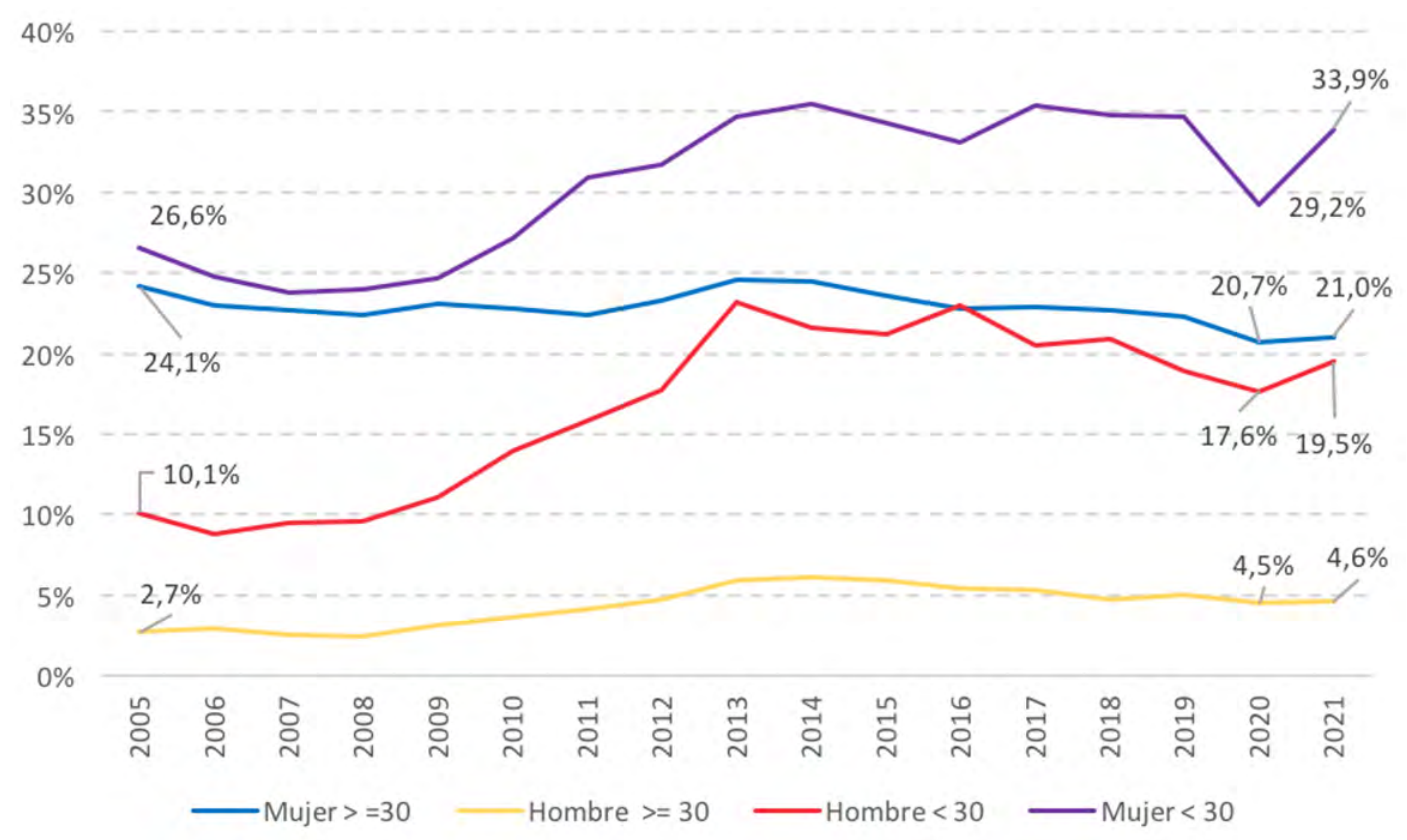

Figura 3. Porcentaje de trabajadores a tiempo parcial por género y grupo de edad.

Fuente: Elaboración propia a partir de datos de EPA 2005-2020 (T2) y 2021 (T1)

En términos globales, España tiene un porcentaje de población empleada a tiempo parcial moderado (14,6\%), muy por debajo de otros países como Holanda (50,2\%), Alemania $(27 \%)$ o Noruega (25,8\%) (datos de Eurostat 2019). Lo que nos diferencia de estos países es el carácter involuntario que predomina en nuestro país. El trabajo a tiempo parcial puede clasificarse como voluntario o involuntario en función de si las personas eligen libremente trabajar menos de 30 horas a la semana o si lo hacen porque es la única opción disponible para ellos en un momento determinado. Así, trabajar a tiempo parcial puede considerarse beneficioso y preferible para los empleados cuando, por ejemplo, necesitan flexibilidad por cuestiones personales (Green y Livanos 2017). Sin embargo, puede convertirse en involuntario cuando el mercado de trabajo no es capaz de adaptar las preferencias de los trabajadores a las necesidades de las instituciones, lo que conduce a una distribución ineficiente de las competencias y los recursos (Green y Livanos 2017). En Espańa, el 55,43\% de los trabajadores a tiempo parcial está insatisfecho por no trabajar a tiempo completo (Eurostat 2019). En el otro extremo se encuentran los Países Bajos, donde sólo el 5,15\% de trabajadores a tiempo parcial lo hace de forma involuntaria.

La tabla a continuación muestra los principales motivos por lo que la población joven, hombres y mujeres, trabajan a tiempo parcial. Aproximadamente la mitad de la población joven con empleo trabaja a tiempo parcial porque no encuentran un trabajo a tiempo completo. Esta cifra es algo superior en el caso de las mujeres, que llega casi al 55\%, mientras que los hombres se sitúan en torno al 51-52\%. Las cifras son similares a las de la población general y no se observan diferencias significativas entre el período pre-covid (2019) y post-covid (2021). El segundo motivo más argüido para trabajar a tiempo parcial es continuar los estudios. El resto lo hacen por otros 
motivos, entre los que se incluye el cuidado de niños o mayores enfermos, otras obligaciones familiares y motivos de salud.

\begin{tabular}{|l|c|c|c|c|}
\hline & \multicolumn{2}{|c|}{ Hombre } & \multicolumn{2}{c|}{ Mujeres } \\
\hline & $\mathbf{2 0 1 9}$ & $\mathbf{2 0 2 1}$ & $\mathbf{2 0 1 9}$ & $\mathbf{2 0 2 1}$ \\
\hline Continuar con los estudios & 34,16 & 33,7 & 28,27 & 29,15 \\
\hline No ha encontrado un trabajo a tiempo completo & 51,65 & 51,27 & 54,55 & 54,71 \\
\hline Otros motivos & 14,19 & 15,03 & 17,18 & 16,14 \\
\hline N & 864 & 632 & 1298 & 892 \\
\hline
\end{tabular}

Tabla 1. Razones por las que tienen contratos a tiempo parcial

Fuente: Elaboración propia a partir de datos de EPA 2005-2020 (T2) y 2021 (T1)

Las altas tasas de trabajo a tiempo parcial involuntario tienen implicaciones importantes, tanto a nivel organizativo como profesional y personal. Desde el punto de vista organizativo, las personas que trabajan menos horas de las que desean muestran menor compromiso afectivo y satisfacción en el trabajo, pudiendo desencadenarse con ello comportamientos laborales que perjudican la productividad, como el absentismo, la impuntualidad, el mal uso del tiempo de enfermedad y otras formas de evasión (Van Emmerik y Sanders 2005, Clark 2001). En el plano profesional, trabajar un número insuficiente de horas está asociado negativamente con los ingresos y con la acumulación de capital humano específico necesario para ser competitivo en el mercado de trabajo y poder negociar una posición mejor. Este es un problema particularmente importante para quienes ocupan puestos en ocupaciones directivas y profesionales, donde las habilidades específicas tienen mayor peso y la formación en el puesto de trabajo resulta imprescindible para promocionar (Budig y England 2001). Por último, el desajuste entre horas deseadas y trabajadas tienen importantes consecuencias para el bienestar personal. A este respecto, las investigaciones señalan que las personas que trabajan menos horas de las que desearían son propensas a tener una menor autoestima (Prause y Dooley 1997). Además, al igual que otras formas de precariedad ya mencionadas, puede provocar retrasos en la edad de emancipación y la postergación, incluso renuncia, de la maternidad (Torre 2020). Por un último, es importante tener en cuenta que este no es sólo un problema de cantidad, sino de intensidad. Entender la magnitud el asunto requiere tomar en consideración el tamaño de la brecha entre horas trabajadas y deseadas, porque desear trabajar 3 horas más por semana no tiene las mismas implicaciones que querer trabajar 10, 15 o 20 horas más. De acuerdo con un estudio reciente llevado a cabo por el Observatorio Social de "la Caixa", el déficit de horas trabajadas pasó de 13,3 en el año 2000, a casi 18 en 2018 (2020).

¿En qué tipo de ocupaciones prolifera la escasez de horas de horas de trabajo? ¿Qué formas de empleo aumentan la brecha entre las horas reales de trabajo y las horas deseadas? La Tabla 2 a continuación muestra el efecto marginal del tipo de ocupación y la modalidad de contrato en la probabilidad de querer trabajar más horas, una vez descontados los efectos sociodemográficos como el género, edad y nivel educativo y nacionalidad. Los trabajadores manuales, o de cuello azul, tienen un $15,2 \%$ más de probabilidad de desear trabajar más horas que quienes están empleados en ocupaciones de cuello blanco - directivos y profesionales. En las ocupaciones administrativas o relacionadas con el sector servicios, también conocidas como ocupaciones de cuello rosa por su alta concentración de mujeres (Howe 1977) la probabilidad es 5,5\% superior que en las de clase alta. La composición de género de las ocupaciones también es un factor determinante a la hora de explicar la falta de trabajo. En coherencia con lo visto en la Figura 3, en las ocupaciones típicamente femeninas (aquellas en las que más del 66\% de las trabajadoras son mujeres) la probabilidad de querer trabajar más horas en un 6\% que en las masculinas (menos del 33\% de mujeres). Estas diferencias 
de género en las horas de trabajo remunerado se traducen en diferencias de género en la experiencia, los ingresos y las oportunidades de promoción futuras (Budig y England 2001). La diferencia entre ocupaciones masculinas y neutras es menor $(2 \%)$ pero aún estadísticamente significativa. En lo que se refiere a la situación contractual, existe una diferencia de 8 por ciento entre jóvenes con contrato temporal e indefinido, aunque la mayor diferencia se encuentra en los que tiene contrato parcial. Tener un empleo a tiempo parcial incrementa un 36\% la probabilidad de querer más horas frente a quienes tienen un contrato indefinido. Las desventajas, pues, son acumulativas.

\begin{tabular}{|c|c|}
\hline & Incremento en la probabilidad de querer más horas \\
\hline \multicolumn{2}{|l|}{ Ocupaciones directivas y profesionales: } \\
\hline Ocupaciones administrativas y de sector servicios & $5,5 \%$ \\
\hline Ocupaciones manuales & $15,2 \%$ \\
\hline \multicolumn{2}{|l|}{ Ocupación masculina (<33\% mujeres): } \\
\hline Ocupación neutra (33\% - 66\% mujeres) & $2,0 \%$ \\
\hline Ocupación femenina (> 66\% mujeres) & $6,0 \%$ \\
\hline \multicolumn{2}{|l|}{ Contrato indefinido: } \\
\hline Temporal & $8,0 \%$ \\
\hline \multicolumn{2}{|l|}{ Contrato a tiempo completo: } \\
\hline Tiempo parcial & $36 \%$ \\
\hline
\end{tabular}

Tabla 2. Efecto marginal de las características ocupacionales y contractuales en la probabilidad de querer trabajar más horas *. Asalariados menores de 30 años.

* Modelo de regresión logística. Incluye controles por edad, nivel educativo, nacionalidad, estatus ocupacional, composición de género de la ocupación, tipo de contrato y tipo de jornada laboral.

Fuente: Elaboración propia a partir de datos de EPA 2021 (T1).

\section{El impacto de la covid-19 en la población joven trabajadora}

Según un sondeo llevado a cabo por la Fundación Europea de Estudios Progresistas en el año 2018, más del $60 \%$ de los encuestados cree que los jóvenes tendrán una vida peor a la de sus padres. La encuesta, además, revelaba la existencia de una brecha entre la Generación X (nacidos entre 1961 y 1981) y la Generación Y (nacidos entre 1981 y 2000, más conocidos como millennials). Cuando se les preguntó sobre el futuro del mercado de trabajo los primeros se mostraron esperanzados, mientras que los últimos se mostraron más temerosos. De hecho, la generación millennial fue la única que no se señaló a sí misma al responder "quién ha vivido/vivirá mejor". Independiente de si esta autopercepción se corresponde o no con la realidad, la presencia de un temor sobre el futuro puede tener un impacto real y negativo en su rendimiento profesional, así como en su salud física y mental (Richter et al. 2020).

El Informe Juventud en España 2020 (INJUVE 2020) señala que uno de cada cuatro jóvenes ve muy probable perder su trabajo en un plazo 12 meses, y uno de cada tres jóvenes en búsqueda activa de empleo no cree que vaya a encontrarlo. Las cifras corresponden al año 2019 y dan cuenta del grado de inseguridad con el que la generación joven se ha enfrentado la nueva crisis, acorralados por la temporalidad y el empleo a tiempo parcial. La visión más pesimista la tenían las mujeres, los y las jóvenes con nivel educativo más bajo y quienes estaban empleados en los sectores más precarios ¿Cuál ha sido el impacto de emergencia sanitaria en la situación laboral de los jóvenes? ¿Cómo la han resistido? Entre el 7 y el 23 de julio de 2020, el Instituto de la Juventud (INJUVE) llevó a cabo un sondeo para evaluar el impacto de confinamiento en la población joven. El estudio consistió en 
1.202 entrevistas telefónicas a jóvenes entre 16 y 30 años con el objetivo de examinar cómo ha afectado el confinamiento a su situación vital, incluyendo su realidad laboral. En la Tabla 3 se recoge el efecto del confinamiento en el trabajo diferenciando por género y estatus ocupacional. De acuerdo con los resultados en Tabla 3, algo más del 16\% de jóvenes ha perdido su empleo durante la etapa de confinamiento. Esta cifra es significativamente mayor para las mujeres $(18,7 \%)$ que para los hombres $(13,3 \%)$ y entre los trabajadores de clase media y baja (18,6\% y $18,5 \%$, respectivamente) que entre los de clase alta $(10,1 \%)$. Este patrón se repite entre los afectados por los ERTE. En general, 6 de cada 10 jóvenes en puestos de alto estatus (directivos y profesionales) no se han visto afectados por el confinamiento, frente al $40 \%$ de sus homólogos en ocupaciones de clase media y baja.

\begin{tabular}{|l|c|c|c|c|c|c|}
\hline & & \multicolumn{2}{|c|}{ Género } & \multicolumn{3}{|c|}{ Estatus ocupacional } \\
\hline & Todos & Mujer & Hombre & $\begin{array}{c}\text { Ocupaciones } \\
\text { Directivos y } \\
\text { profesionales } \\
\text { administrativas } \\
\text { y del sector } \\
\text { servicios }\end{array}$ & $\begin{array}{c}\text { Ocupaciones } \\
\text { manuales }\end{array}$ \\
\hline No me ha afectado & 47,7 & 45,7 & 50,0 & 63,1 & 40,2 & 44,4 \\
\hline He hecho uso de mis días de vacaciones & 1,5 & 1,6 & 1,4 & 1,2 & 1,4 & 2,8 \\
\hline Me han aplicado un ERTE & 24,1 & 26,4 & 21,6 & 13,7 & 30,4 & 25,0 \\
\hline Soy autónomo/a y he cesado mi actividad & 3,9 & 2,9 & 5,0 & 3,6 & 3,7 & 3,7 \\
\hline He perdido el empleo & 16,2 & 18,7 & 13,3 & 10,1 & 18,6 & 18,5 \\
\hline Otros & 6,6 & 4,8 & 8,6 & 8,3 & 5,7 & 5,6 \\
\hline
\end{tabular}

Tabla 3: Cómo ha afectado el confinamiento a la situación laboral de los jóvenes.

Fuente: Elaboración propia a partir del Sondeo covid-19 (INJUVE 2020)

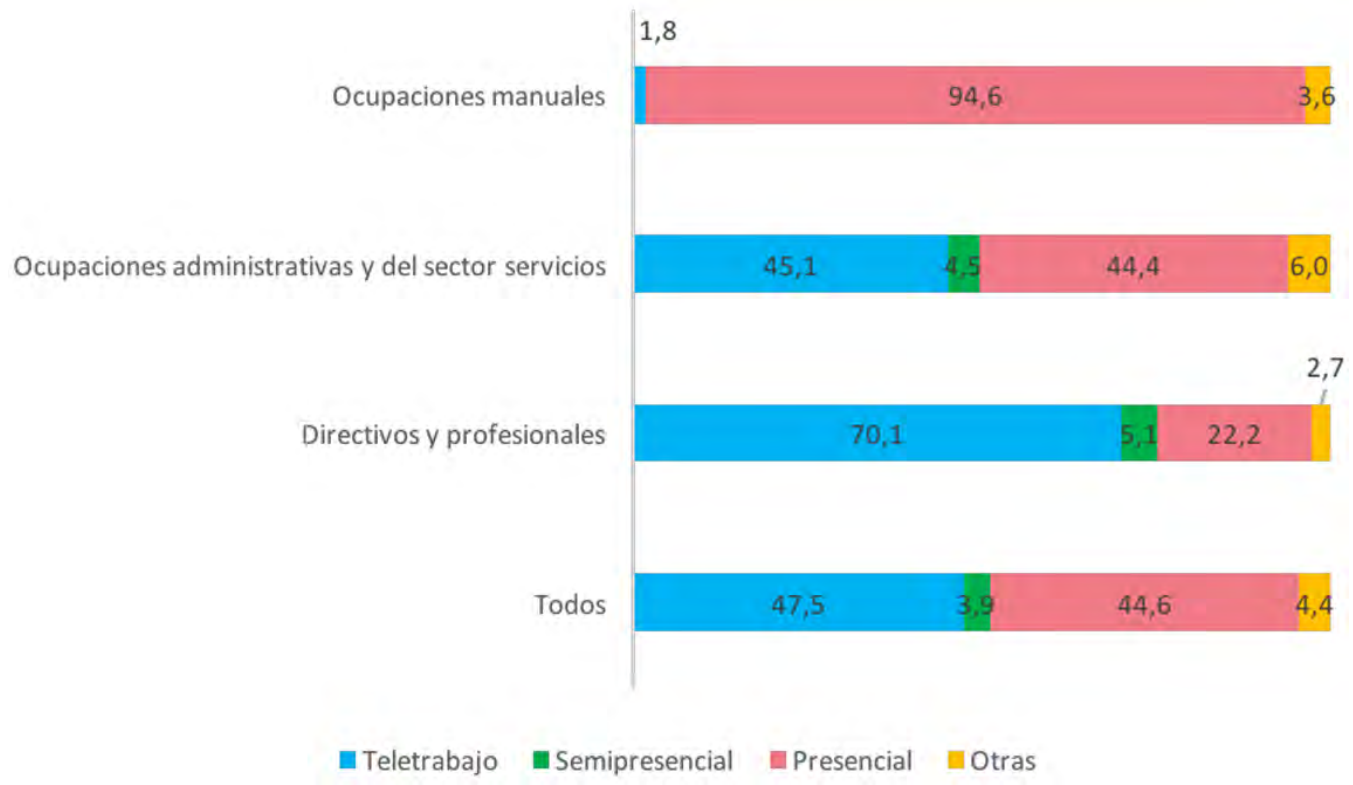

Figura 4. Modalidad de empleo durante el confinamiento.

Fuente: Elaboración propia a partir de la Encuesta Covid-19 INJUVE 2020.

Las diferencias de clase en la pérdida de empleo están, en gran medida, relacionadas con la modalidad de trabajo durante el confinamiento. De acuerdo con la Figura 4, el teletrabajo ha sido la modalidad predominante entre quienes ocupan puesto de alto estatus (directivos y profesionales). 
Esta modalidad no sólo previene el riesgo de empleo, sino que es la que reduce en mayor medida el riesgo de contagio (Gascon 2020). Entre las ocupaciones administrativas y del sector servicios el trabajo se ha repartido entre modalidad presencial y teletrabajo, mientras que para los trabajadores manuales el teletrabajo no es una opción. En consecuencia, los empleados de clase baja no sólo se ha perdido más empleo, como se veía en la Tabla 3, sino que se han visto más expuestos a una situación de riesgo para su salud.

En general, los datos confirman qua crisis COVID-19 está teniendo un mayor impacto en algunos trabajadores que en otros. Las mujeres y los trabajadores precarios se encuentran entre los que corren mayor riesgo de desempleo y pobreza. Suelen tener empleos menos seguros y no cualificados y están muy representados entre los trabajadores de los sectores más afectados por la crisis, como el turismo y la restauración. Desde el punto de vista sanitario, también han sido los más expuestos, puesto que a menudo sus ocupaciones se han considerado esenciales.

\section{Reflexiones finales: Un problema estructural que requiere problemas estructurales}

La recesión económica provocada por la COVID-19 ha cristalizado la espiral de precariedad y falta de empleo juvenil que se había iniciado con la crisis del 2008. Cada vez más, los jóvenes se enfrentan a dificultades para encontrar y mantener empleos de calidad, y la brecha intergeneracional se engrosa. Los datos, además, apuntan a un incremento de las diferencias intrageneracionales, con los trabajadores de bajo y medio estatus más propensos a sufrir más temporalidad y un déficit de horas de trabajo que no garantiza un sueldo mínimo necesario para emanciparse y formar una familia.

Alcanzar mayores niveles educativos resulta, sin duda, un eficaz protector frente al riesgo de desempleo y un potente catalizador de la clase social (Requena 2016, OECD 2015). Sin embargo, mejorar el acceso a la educación terciaria es insuficiente para erradicar la desigualdad y precariedad laboral entre los jóvenes. El problema del trabajo precario obedece a causas estructurales y, en consecuencia, necesita soluciones estructurales. Prueba de ello es la sobreeducación continua presente en la realidad laboral de nuestro país (Pascual Sáez et al. 2015). La Figura 5 muestra la distribución de jóvenes universitario y no universitarios según estatus ocupacional (valor promedio 2005-2021). Casi un 40\% de

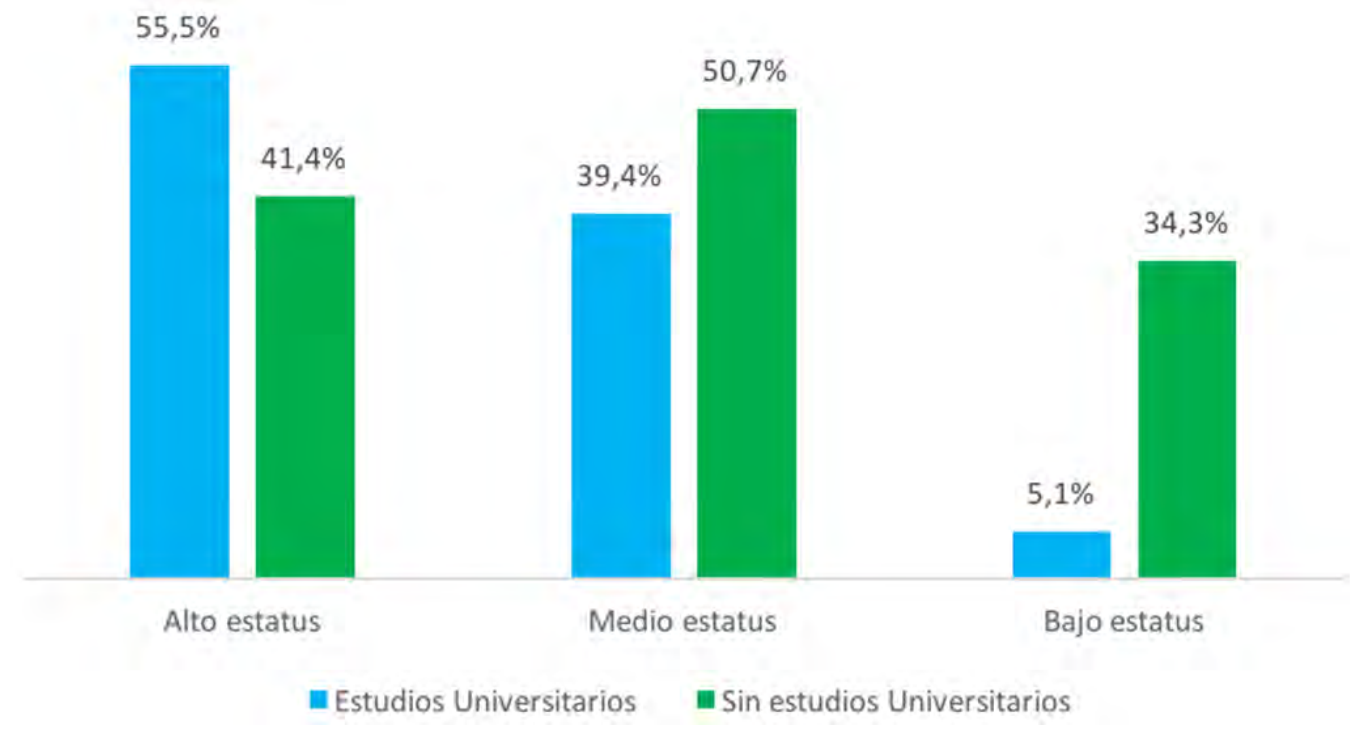

Figura 5. Población joven con y sin título universitario según estatus ocupacional. Promedio 2005-2021. Fuente: Elaboración propia a partir de datos de EPA 2005-2020 (T2) y 2021 (T1). 
la población joven con estudios superiores ocupa puestos administrativos o relacionados con el sector servicios como, por ejemplo, administrativos sin tareas de atención al público, agentes y representantes comerciales y camareros/as asalariados. Un 5\% adicional trabaja en ocupaciones manuales poco cualificadas, principalmente como personal de limpieza de oficinas, hoteles y otros establecimientos similares, empleadas domésticas, peones de la construcción y de la minería y reponedores.

Andreas Schleicher, creador del informe PISA, denunciaba recientemente en una entrevista concedida a el periódico El País la dificultad de los jóvenes españoles con titulaciones universitarias avanzadas para encontrar un buen trabajo (Zafra 2021). El experto resaltaba que, paradójicamente, los empleadores españoles dicen que no encuentran a las personas con las habilidades que necesitan. En otras palabras, el sistema educativo fracasa a la hora de formar para las nuevas necesidades.

La solución pasa por acometer reformas estructurales tanto del mercado de trabajo como del binomio trabajo-escuela: reducir la temporalidad en el empleo, garantizar las horas de trabajado necesarias y (re)diseñar el sistema educativo para garantizar el ajuste entre las habilidades adquiridas y las habilidades demandadas por el mercado de trabajo. Además, es importante reorientar las actuaciones del estado de bienestar para cumplir con el principio de justicia intergeneracional, garantizar el bienestar de las generaciones jóvenes y contribuir a reducir la brecha de clase social.

\section{Bibliografía}

BERRY, C.; McDaniel, S., "Post-crisis precarity: Understanding attitudes to work and industrial relations among young people in the UK". Economic and Industrial Democracy, 2020

CABRALES, A.; DOLADO, J.J.; MORA, R., "Dual employment protection and (lack of) on-thejob training: PIAAC evidence for Spain and other European countries" SERIEs 8, 345-371. 2017. https://doi.org/10.1007/s13209-017-0166-9

CHINCHILLA, N.; JIMÉNEZ, E.; GRAU, M., "Maternidad y trayectoria profesional en España: análisis de las barreras e impulsores para la maternidad de las mujeres españolas", IESE Business School-ORDESA (ST-444), 2017

CLARK, A. E., "What really matters in a job? Hedonic measurement using quit data", Labor Economics, 8, 2001

DOOLEY, D., PRAUSE, J.; HAM-ROWBOTTOM, K. A., “Underemployment and depression: longitudinal relationships", Journal of Health and Social Behavior, 41, 2000

EUROSTAT. Euroindicators. 2021. https://ec.europa.eu/eurostat/documents/2995521/11563067/330042021-CP-EN.pdf/5e5aae01-e15d-b8bd-71fb-4096b88f4120?t=1619705933576

FERNANDEZ, J.J. "Evolución comparada de la pobreza infantil, juvenil, y de los mayores en Europa", Observatorio Social de "la Caixa". 2017

GASCON, G., "COVID-19: Which workers face the highest unemployment risk?” St. Louis Federal on the Economy, March 232020.

GREEN, A. E.; LIVANOS, I., "Involuntary non-standard employment in Europe". European Urban and Regional Studies, 24 (2): 175-192. 2017

HOWE, L. K., "Pink collar workers: inside the world of women's work". New York: Putnam. 1977

JACOBS, J.A., GERSON, K., "The time divide: work, family, and gender inequality", Cambridge, MA: Harvard university press. 2001

OECD, "Education at a Glance Interim Report: Update of employment and educational attainment indicators" París: OECD, 2015. http://www. oecd.org/edu/eag-interim-report.pdf

OECD, "Society at a Glance", 2016. https://doi.org/10.1787/19991290.

OECD, "Youth and Covid-19: Response, recovery and resilience". 2020. https://read.oecd-ilibrary. 
org/view/?ref=134_134356-ud5kox3g26\&title=Youth-and-COVID-19-Response-Recoveryand-Resilience\&_ga=2.186501033.146659613.1624271104-965303261.1624271104

OECDE, "Youth and Covid-19: Response, recovery and resilience", 2020. https://read.oecdilibrary.org/view/?ref=134_134356ud5kox3g26\&title=Youth-and-COVID-19-ResponseRecovery-and-Resilience

PASCUAL SÁEZ, M., GONZÁLEZ-PRIETO, N., CANTARERO-PRIETO, D., "Is Over-Education a Problem in Spain? Empirical Evidence Based on the EU-SILC". Social Indicators Research, 126: 617-632. 2016

PEREZ DEL PRADO, D., "El caldo de cultivo para un pacto social. Algunos datos para reformar el mercado de trabajo en España”, Labos. Revista de Derecho del Trabajo y Protección Social 2(1):137-151. 2021.

REQUENA, M., "El ascensor social. ¿Hasta qué punto una mejor educación garantiza una mejor posición social?", Observatorio Social de "la Caixa". 2016.

REQUENA, M., "Juventud y dependencia familiar en España”, Revista de Estudios de Juventud, 58:19-32. 2002

TORRE, M., "Desajustes entre las horas trabajadas y deseadas en la población joven. Un análisis de la evolución e España, 20000-2018”, El Observario Social, Fundación "la Caixa”. 2020

VAN EMMERIK, I.J.H.; SANDERS. K., "Mismatch in Working Hours and Affective Commitment: Differential Relationship for Distinct Employees Groups." Journal of Managerial Psychology 20(8):712-726. 2005.

ZAFRA, I., (junio 18, 2021). El creador del informe PISA "La educación en Espańa prepara a los alumnos para un mundo que ya no existe". elpais.com https://elpais.com/educacion/2021-06-18/el-creador-del-informe-pisa-la-educacion-espanola-prepara-a-los-alumnos-para-un-mundo-que-ya-no-existe.html 